\title{
Monolithic Integration of a Novel Microfluidic Device with Silicon Light Emitting Diode-Antifuse and Photodetector
}

\author{
P. LeMinh, J. Holleman, \\ J.W. Berenschot, N.R. Tas, A. van den Berg \\ $\mathrm{MESA}^{+}$Research Institute, University of Twente, the Netherlands \\ E-mail P.LeMinh@el.utwente.nl
}

\begin{abstract}
Light emitting diode antifuse has been integrated into a microfluidic device that is realized with extended standard CMOS technological steps. The device comprises of a microchannel sandwiched between a photodiode detector and a nanometer-scale diode antifuse light emitter. Within this contribution, the device fabrication process, working principle and properties will be discussed. Change in the interference fringe of the antifuse spectra has been measured due to the filling of the channel. Preliminary applications are electroosmotic flow speed measurement, detection of absorptivity of liquids in the channel...
\end{abstract}

\section{Introduction}

Silicon is well-known as an electronic and mechanical material but hardly ever used as an active optoelectronic element, due to its inefficient radiative recombination over an indirect bandgap. Integrated silicon optical sensors themselves encounter that critical obstacle. Recently, silicon nanometer-scale diode antifuse has emerged as a reasonable choice with improved efficiency, small size, and CMOS compatible fabrication[1][2].

With the rapid growth of microfluidic and microchemical systems in research as well as in applications, specialized devices for sensing and measuring parameters are becoming more and more important [6][7]. The integration of the light emitter and detector with a microfluid channel provides such a universal tool for that purpose. This device structure has also been greatly desired in $\mu$ TAS, biochemical sensors and actuators[4][5]. Furthermore, the approach can also give birth to new types of sensors.

The technology used in the creation of this device is a combination of standard CMOS technology with silicon sensor micromachining technology. The antifuse and photodiode were made by standard CMOS steps, but the microchannel required a sacrificial poly-Si etch in potassium hydroxide $(\mathrm{KOH})$. However, we are now developing an CMOS compatible etching recipe using Tetra Methyl Ammonium Hydroxide (TMAH).

In the next sections of the paper, fabrication process, some critical technological design parameters, electrical and optical properties, few results on first applications will be described respectively.

\section{Device realization}

The realization of the device (figure 1) is a 10-mask process. The starting material is a 3 -in silicon n-type wafer with standard doping. First of all, junction photodiode areas were fabricated by implantation of boron. So as not to make any step height difference at the junction between n-type and p-type region, thick resist was used as masking layer for the implantation. This is important to the evenness of the channel. The shape of the channel was then produced by shaping a layer of 500nm thick LPCVD polysilicon deposited on top of the first silicon rich nitride ( $\mathrm{SiRN}$ ) layer. Next another SiRN film is deposited to completely cover the polysilicon. These two SiRN layers acts as walls of the channel opened later by sacrificial polysilicon etching in $\mathrm{KOH}$. They also electrically isolate the detector and integrated antifuse from the channel. On top of the second SiRN layer, a capacitor structure with size of $5 \times 5 \mu \mathrm{m}^{2}$ is fabricated. The first electrode is $300 \mathrm{~nm}$ phosphorus implanted polysilicon. The capacitor dielectric layer is 8nm LPCVD deposited silicon dioxide. The second polysilicon electrode was implanted with $\mathrm{BF}_{2}$ with a dose of $3.10^{15} \mathrm{~cm}^{-2}$ at $70 \mathrm{keV}$ (figure 2,3). The activation of all dopants were simultaneously done in the next step as a protection layer of $\mathrm{SiRN}$ was deposited at $850^{\circ} \mathrm{C}$ for approximately $90 \mathrm{~min}$. Next, for the opening of the channel, windows at two channel ends were patterned and etched until reaching the sacrificial polysilicon. The etching in $\mathrm{KOH}$ etches polysilicon at a rate of roughly $1 \mu \mathrm{m}$ per minute. That means a channel of $2 \mathrm{~mm}$ would take almost 1.5 days of etching (figure 4) [3]. The silicon wafer is, however, well protected because the SiRN is etched extremely slow in $\mathrm{KOH}$. SiRN was also chosen for the process due to its low mechanical stress compared to stoichiometric silicon nitride. As the channel was 
etched through, the processed wafer then needed a standard treatment to dissolve alkali ions and other contaminations. The inner surfaces of the channel needed hydrophilic "Piranha" treatments to be able to conduct liquid. The next step is to make electrical contacts to the detector and the antifuse by SiRN etching and metal deposition. To fill the channel, confinement of liquid at the channel ends is necessary. This is done by plasma deposition of a hydrophobic FC-layer in the form of rings onto the wafer using a shadow mask. The process was finished with the etching and metalization of the back side.

\section{Device properties and discussion}

The completion of the device showed that a microfluidic component could be integrated into standard CMOS device structure (figure 1, 2, 3, 4). The device strategy is vertical instead of lateral [7]. The novelty of this structure proves that waveguide is not always necessary. By the geometry, it is clear that coupling loss and loss within the fibre and can be avoided.

In figure 5, the pre-breakdown current voltage characteristics of the antifuse capacitor are shown in both biasing polarities. It shows well known Fowler-Nordheim tunnelling at the applied electrical field of approximately $10 \mathrm{MVcm}^{-1}$. At lower field, the leakage current shows pattern as has been commonly observed in standard thin oxide MOS capacitors.

In order to create the antifuse, a constant current of $100 \mathrm{nA}$ was used to stress the capacitor until breakdown (known as charge to breakdown method) in electron injection mode. Further programming currents up to $5 \mathrm{~mA}$ were then applied. The diode antifuse emits visible light in reverse breakdown condition. Current voltage curve of the antifuse is plotted in figure 6 showing a breakdown voltage at $3 \mathrm{~V}$. The mechanism of the breakdown is supposed to be Zener breakdown due to the highly-doped level of both electrodes. The series resistance, has not been improved from the device reported in [1], is largely caused by the columnar structure of the polysilicon (high sheet resistance). For a better power conversion efficiency of the emitter, the future device should be fabricated with small grain polysilicon.

In figure 7 , the spectra at two emitting currents are displayed showing the interference fringes of the emitted light waves due to the multi-layer structure of the device. The fringes have been previously observed in simple poly/mono antifuse structure. In this case, the pattern is more complicated with more pronounced peaks and valleys. Nevertheless, the position of these extremes could be altered by filling the channel with other materials instead of air. The displacement of the extremes is dependent on the complex refractive index of the filled material and can be theoretically calculated by multi-layer physical optics formulations.
We have filled the channel with deionised water and aqueous fluorescine solution, measured and compared the spectra at $1 \mathrm{~mA}$ to the one of unfilled channel. The shift of the extremes is clearly seen as shown in figure 8 . The measurements on the unfilled channel was done both before and after the liquid filling, showing consistent shape and slope. It is clear that this device has potential applications in sensing metrology for microfluidics.

The mechanism of flow speed measurement and absorptivity can be discussed based on figure 9 whereby showing the photocurrents dependence on the regulated antifuse currents [1]. At a fixed light emitting current, the change in the medium of the channel can be sensed on the detector by the alteration in the magnitude of the photocurrent. This alteration is caused by the absorption of photon in the liquid that fills the channel, and the new refractive index difference at the two interfaces of the channel walls.

\section{Conclusion}

The success of the novel device indicates that a system of light emitter, photodetector and a reaction chamber, a microchannel in this case, can be fabricated on silicon with CMOS technology. For the first time, nanometer-scale light emitting diode antifuse has been applied to a microfluidic device. The electrical and optical properties of the components are signs of a newly proven technological combination and realistic device working principle.

\section{Acknowledgement}

We acknowledge the assistance of M. de Boer and A. Aarnink. This work is supported by MiCS, a strategic research orientation at University of Twente.

\section{References}

[1]. P. LeMinh et al., "A Novel Silicon Electro-Optical Device for Sensor Applications", Proc. IEEE/ LEOS 2000, Puerto Rico, 2000, pp. 523-524.

[2]. P. LeMinh et al., "Photochemical Reaction By Nanometer-scale Light Emitting Diode-Antifuses", Proc. Transducer'01, Munich 2001, pp. 544-547.

[3]. J.W. Berenschot et al., "Advanced Sacrificial Poly-Si Technology for Fluidic Systems", Proc. Transducer '01, Munich 2001, pp. 624-627.

[4]. A. van den Berg et al., "Micro Total Analysis Systems: Microfluidic Aspects, Integration Concept and Applications" Topics Cur. Chem., 194, (1997), pp. 21-50.

[5]. Yu-Chong Tai, Presentation "MEMS Sensors for Biomedical Applications" NSF/ERC Industry Day, May 16-17, 2001, California Institute of Technology.

[6]. N. R. Tas et al., "Nanofluidic bubble pump using surface tension directed gas injection" accepted for Analytical Chemistry, 2002.

[7]. M. Foquet et al., "DNA fragment sizing by single molecule detection in submicrometer-sized closed fluidic channels" accepted for Analytical Chemistry, 2002. 


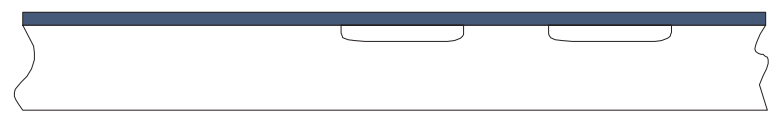

Photodetector \& $1^{\text {st }}$ SiRN

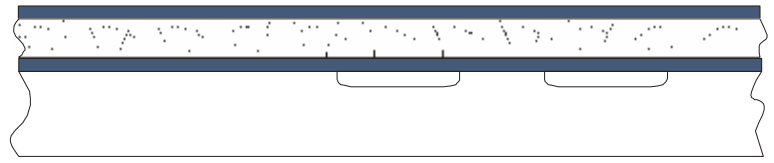

Poly-Si channel $\& 2^{\text {nd }}$ SiRN

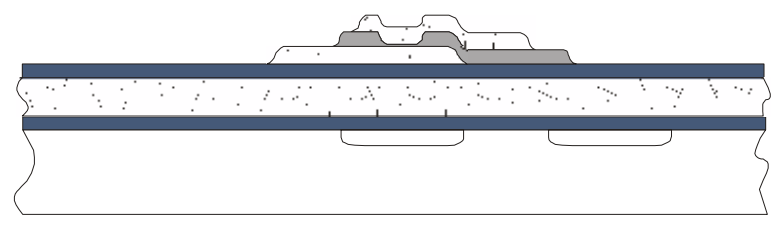

Antifuse structure on $2^{\text {nd }}$ SiRN

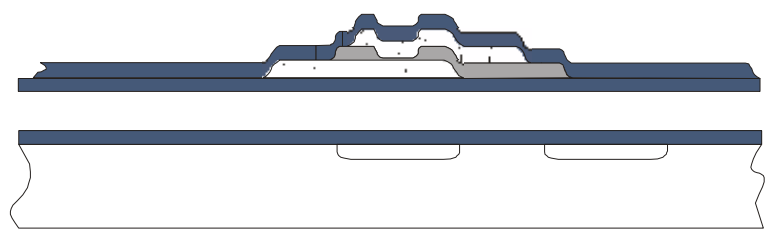

$3^{\text {rd }}$ SiRN cover \& channel openning

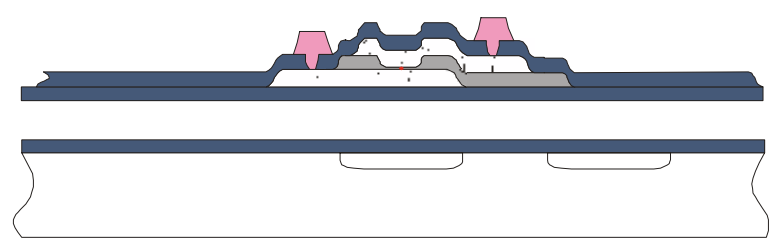

\section{Metal contacts}

Figure 1. Device fabrication process

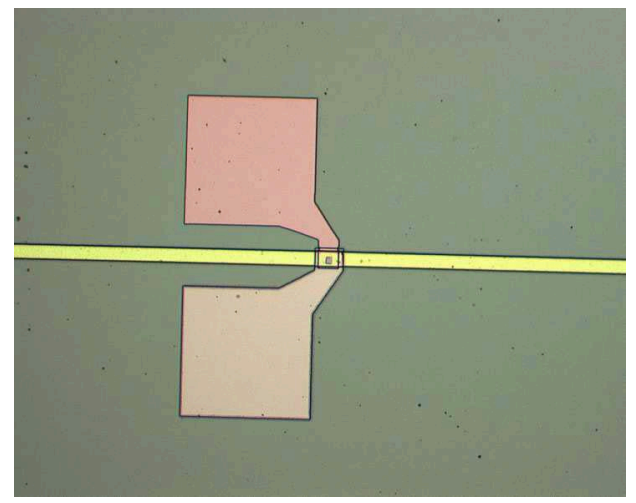

Figure 2. Channel under the antifuse window, two polysilicon electrodes are clearly visible

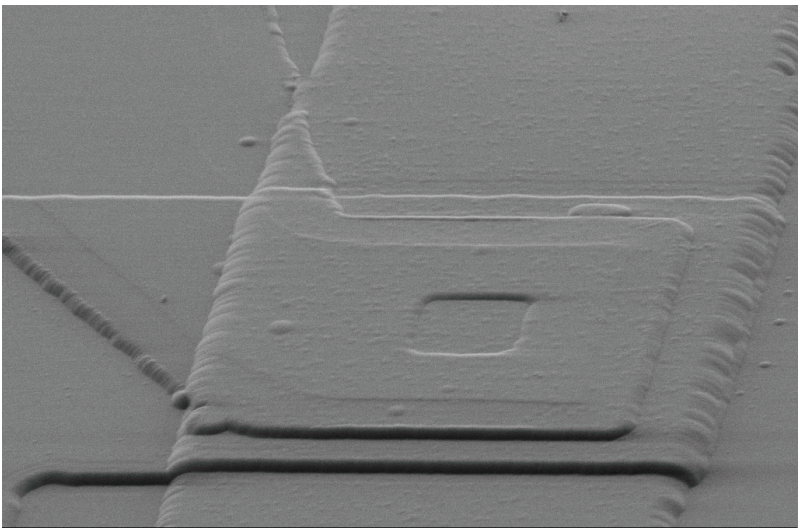

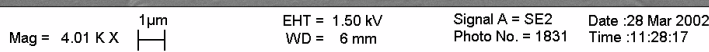

Figure 3. SEM image of integrated device on top of the channel

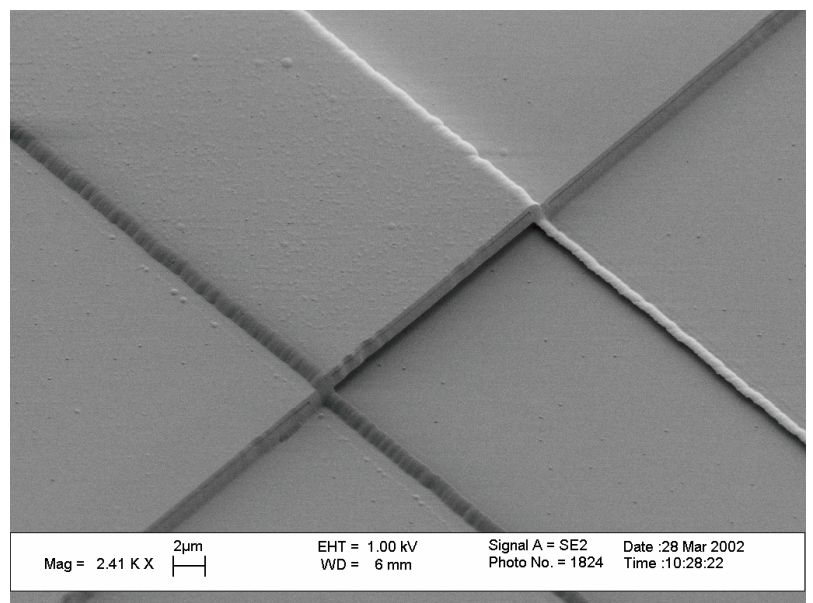

Figure 4. SEM image of channel entrance

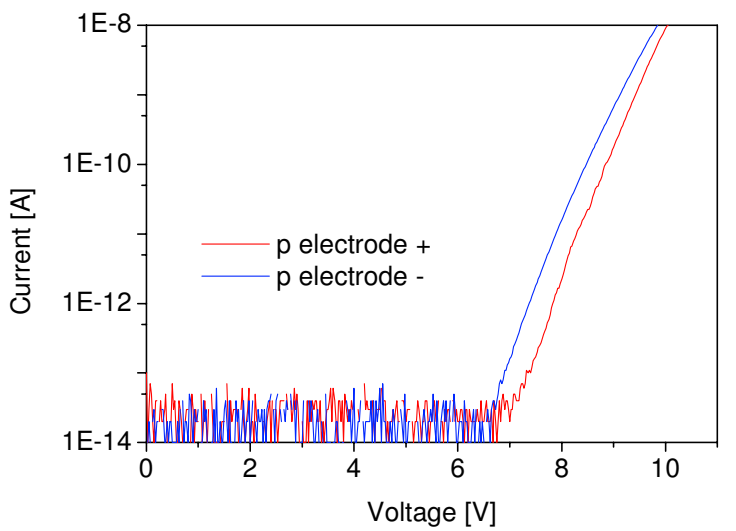

Figure 5. Pre-breakdown current voltage curve of the antifuse capacitor in both biasing directions 


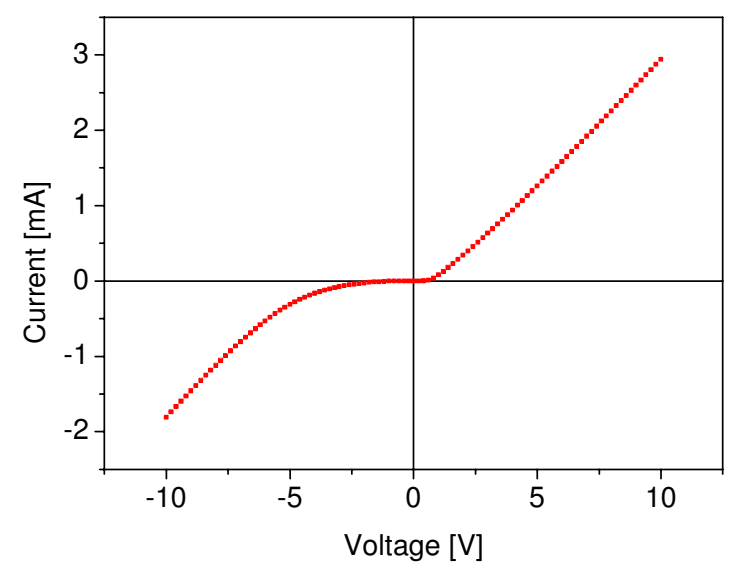

Figure 6. Current voltage characteristics of the diode antifuse

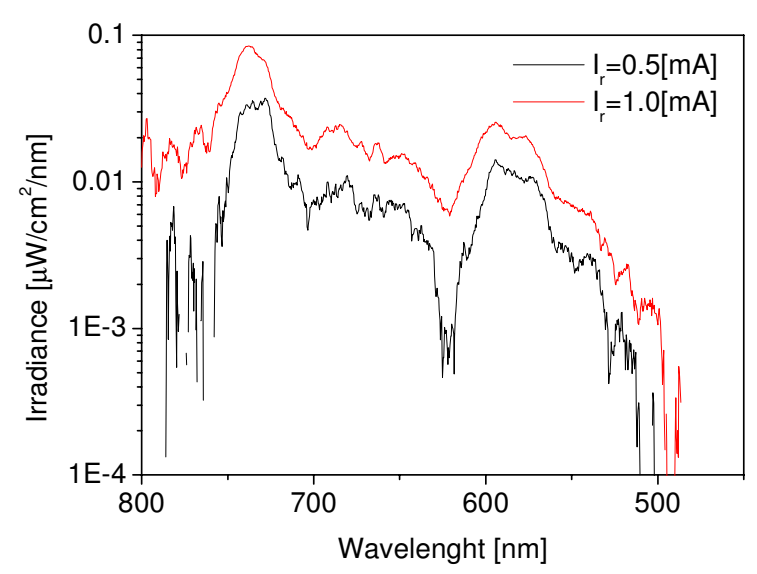

Figure 7. Irradiance spectra of the integrated antifuses

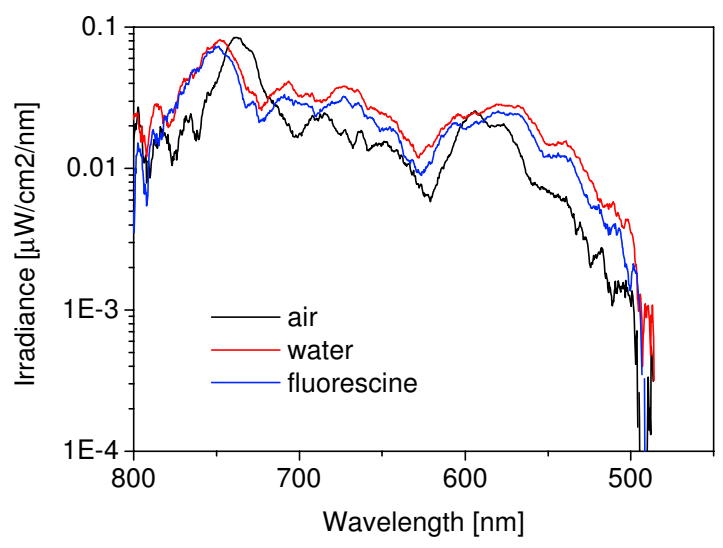

Figure 8. Filled channels shifting the interference pattern of the antifuse spectra at $I=1 \mathrm{~mA}$

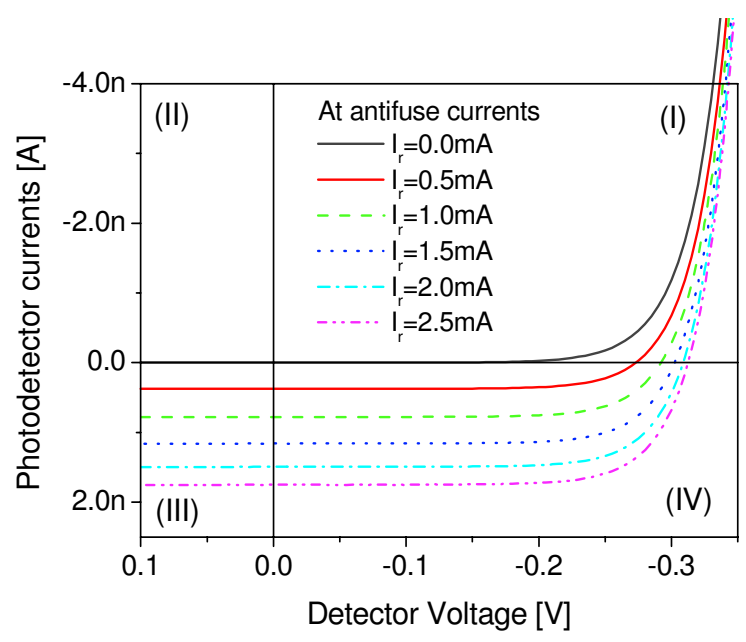

Figure 9. Photocurrents on the detector 